\title{
Floor-Planning via Orderly Spanning Trees ${ }^{\star}$
}

\author{
Chien-Chih Liao ${ }^{1}$, Hsueh-I. Lu ${ }^{2}$, and Hsu-Chun Yen ${ }^{3}$ \\ 1 Department of Electrical Engineering, National Taiwan University \\ Taipei 106, Taiwan, Republic of China \\ henry@cobra.ee.ntu.edu.tw \\ 2 Institute of Information Science, Academia Sinica \\ Taipei 115, Taiwan, Republic of China \\ hil@iis.sinica.edu.tw \\ http://www.iis.sinica.edu.tw/ ${ }^{\sim}$ hil \\ ${ }^{3}$ Department of Electrical Engineering, National Taiwan University \\ Taipei 106, Taiwan, Republic of China \\ yen@cc.ee.ntu.edu.tw \\ http://www.ee.ntu.edu.tw/ yen
}

\begin{abstract}
Floor-planning is a fundamental step in VLSI chip design. Based upon the concept of orderly spanning trees, we present a simple $O(n)$-time algorithm to construct a floor-plan for any $n$-node plane triangulation. In comparison with previous floor-planning algorithms in the literature, our solution is not only simpler in the algorithm itself, but also produces floor-plans which require fewer module types. An equally important aspect of our new algorithm lies in its ability to fit the floor-plan area in a rectangle of size $(n-1) \times\left\lfloor\frac{2 n+1}{3}\right\rfloor$.
\end{abstract}

\section{Introduction}

In VLSI chip design, floor-planning [2016] refers to the process of, given a graph whose nodes (respectively, edges) representing functional entities (respectively, interconnections), partitioning a rectangular chip area into a set of nonoverlapping rectilinear polygonal modules (each of which describes a functional entity) in such a way that the modules of adjacent nodes share a common boundary. For example, Figure 1(b) is a floor-plan of the graph in Figure 1(a).

Early stage of the floor-planning research focused on using rectangular modules as the underlying building blocks. A floor-plan using only rectangles to represent nodes is called a rectangular dual. It was shown in 13 14,15] that a plane triangulation $G$ admits a rectangular dual if and only if $G$ has four exterior nodes, and $G$ has no separating triangles. (A separating triangle, which is also known as complex triangle 2120, is a cycle of three edges enclosing some nodes in its interior.) As for floor-planning general plane graphs, Yeap and Sarrafzadeh [21] showed that rectilinear modules with at most two concave corners are sufficient and necessary.

* Research supported in part by NSC Grant 90-2213-E-002-100.

P. Mutzel, M. Jünger, and S. Leipert (Eds.): GD 2001, LNCS 2265, pp. 367-377, 2002.

(C) Springer-Verlag Berlin Heidelberg 2002 


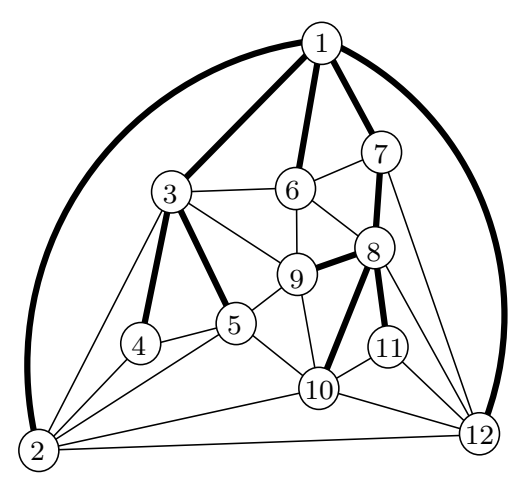

(a)

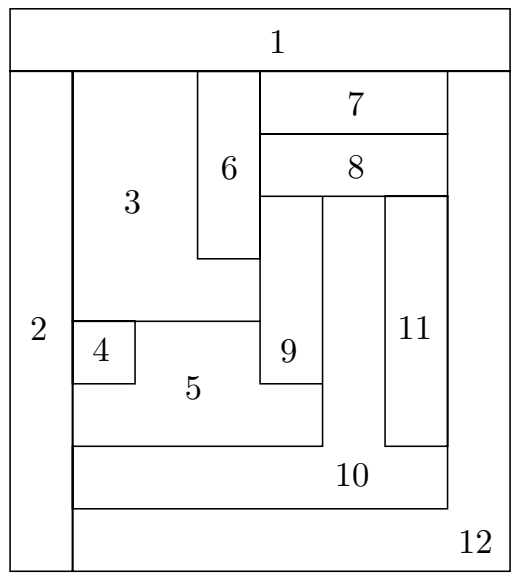

(b)

Fig. 1. (a) A plane triangulation $G$, where an orderly spanning tree $T$ of $G$ rooted at node 1 is drawn in dark. The node labels show the counterclockwise preodering of the nodes in $T$. (b) A floor-plan of $G$.

In a subsequent study of floor-planning, He 9 measured the complexity of a module in terms of the number of its constituent rectangles, as opposed to the amount of concave corners. A module that is a union of $k$ or fewer disjoint rectangles is called a $k$-rectangular module. Since any rectilinear module with at most two concave corners can be constructed by three rectangular modules, the result of Yeap and Sarrafzadeh [21] implies the feasibility of floor-planning plane graphs using 3-rectangular modules. He [9] presented a linear-time algorithm to construct a floor-plan of a plane triangulation using only 2-rectangular modules. He's floor-planning algorithms consists of three phases: The first phase utilizes the canonical ordering 6 11,12 to assign nodes on separating triangles. The second phase involves the so-called vertex expansion operation to break all

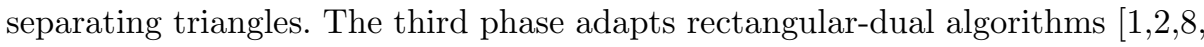
12 to finalize the drawing of the floor-plan. Figure 2 depicts the shapes of the 2 -rectangular modules required by He's algorithm. For convenience, these four shapes are referred to as I-module, L-module, T-module, and Z-module throughout the rest of this paper.

In this paper, we provide a "simpler" linear-time algorithm that computes "compact" floor-plans for plane triangulations. The "compactness" of the output floor-plans is an important advantage of our algorithm over previous results [9, 21. Specifically, the output of our algorithm for an $n$-node plane triangulation has area no more than $(n-1) \times\left\lfloor\frac{2 n+1}{3}\right\rfloor$. Previous work [9,21], however, reveals no such area information. What "simplicity" means is two-fold:

- First, as opposed to the multiple-phase approach of [921], our algorithm is based upon a recent development of orderly spanning trees [3], which provides 


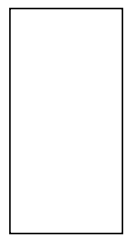

(a)

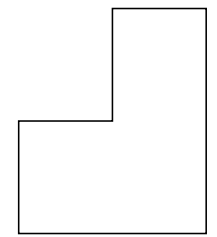

(b)

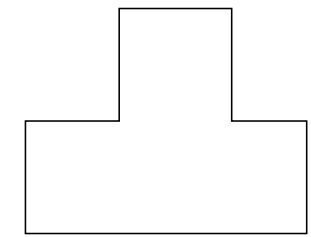

(c)

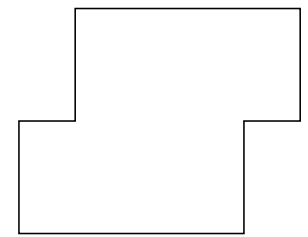

(d)

Fig. 2. Four types of modules required by He's floor-planning algorithm 9]: (a) Imodule, (b) L-module, (c) T-module, and (d) Z-module. Our algorithm does not need Z-modules.

an extension of canonical ordering 61112 to plane graphs not required to be triconnected and an extension for realizer [19 18] to plane graphs not required to be triangulated. Our approach bypasses the somewhat complicated rectangular-dual phase. Aside from the two applications of orderly spanning trees reported in [3] (namely, succinct encodings for planar graphs with efficient query support [10,17,4] and 2-visibility drawings for planar graphs [7]), our investigation here finds another interesting application of orderly spanning trees.

- Second, the floor-plan design of our algorithm is "simpler" (in comparison with [9]) in its own right, in the sense that I-modules, L-modules, and Tmodules suffice. (Recall that Z-modules are needed by He's algorithm 9].)

The remainder of this paper is organized as follows. Section 2 gives the preliminaries. Section 3 presents our linear-time floor-planning algorithm as well as its correctness proof. Section 4 concludes the paper.

\section{Preliminaries}

A plane graph is a planar graph equipped with a fixed planar embedding. The embedding of a plane graph divides the plane into a number of connected regions, each of which is called a face. The unbounded face of $G$ is called the exterior face, whereas the remaining faces are interior faces. $G$ is a plane triangulation if $G$ has at least three nodes and the boundary of each face, including the exterior face, of $G$ is a triangle. Let $T$ be a rooted spanning tree of a plane graph $G$. Two nodes are unrelated in $T$ if they are distinct and neither of them is an ancestor of the other in $T$. An edge of $G$ is unrelated with respect to $T$ if its endpoints are unrelated in $T$. Let $v_{1}, v_{2}, \ldots, v_{n}$ be the counterclockwise preordering of the nodes in $T$. A node $v_{i}$ is orderly in $G$ with respect to $T$ if the neighbors of $v_{i}$ in $G$ form the following four blocks in counterclockwise order around $v_{i}$ :

$B_{1}\left(v_{i}\right)$ : the parent of $v_{i}$

$B_{2}\left(v_{i}\right)$ : the unrelated neighbors $v_{j}$ of $v_{i}$ with $j<i$, 
$B_{3}\left(v_{i}\right)$ : the children of $v_{i}$, and

$B_{4}\left(v_{i}\right)$ : the unrelated neighbors $v_{j}$ of $v_{i}$ with $j>i$,

where each block could be empty. $T$ is an orderly spanning tree of $G$ if $v_{1}$ is on the boundary of $G$ 's exterior face, and each $v_{i}, 1 \leq i \leq n$, is orderly in $G$ with respect to $T$. It is not difficult to see that if $G$ is a plane triangulation, then $B_{2}\left(v_{i}\right)$ (respectively, $B_{4}\left(v_{i}\right)$ ) is nonempty for each $i=3,4, \ldots, n$ (respectively, $i=2,3, \ldots, n-1$. For each $i=2,3, \ldots, n$, let $p(i)$ be the index of the parent of $v_{i}$ in $T$. Let $w(i)$ denote the number of leaves in the subtree of $T$ rooted at $v_{i}$. Let $\ell(i)$ and $r(i)$ be the functions such that $v_{\ell(i)}$ (respectively, $\left.v_{r(i)}\right)$ is the last (respectively, first) neighbor of $v_{i}$ in $B_{2}\left(v_{i}\right)$ (respectively, $\left.B_{4}\left(v_{i}\right)\right)$ in counterclockwise order around $v_{i}$. For example, in the example shown in Figure廿(a), one can easily verify that node 3 is indeed orderly with respect to $T$, where $B_{1}(3)=\{1\}, B_{2}(3)=\{2\}, B_{3}(3)=\{4,5\}, B_{4}(3)=\{6,9\}, p(3)=1, w(3)=2$, $\ell(3)=2$, and $r(3)=9$. When $G$ is a plane triangulation, it is known [3] that for each edge $\left(v_{i}, v_{j}\right)$ of $G-T$ with $i<j$, at least one of $i=\ell(i)$ and $j=r(i)$ holds. To be more specific, if $i=2$ and $j=n$, then both $3=\ell(n)$ and $n=r(3)$ hold; otherwise, precisely one of $i=\ell(i)$ and $j=r(i)$ holds.

The concept of orderly spanning tree for planar graphs [3] extends that of canonical ordering 6|11|12 for plane graphs not required to be triconnected and that of realizer [19]18]5 for plane graphs not required to be triangulated. Specifically, when $G$ is a plane triangulation, (i) if $T$ is an orderly spanning tree of $G$, then the counterclockwise preordering of the nodes of $T$ is always a canonical ordering of $G$, and (ii) if $\left(T_{1}, T_{2}, T_{n}\right)$ is a realizer of $G$, where $T_{i}$ is rooted at $v_{i}$ for each $i=1,2, n$, then each $T_{i}$ plus both external edges of $G$ incident to $v_{i}$ is an orderly spanning tree of $G$. Our floor-planning algorithm is based upon the following lemma.

Lemma 1 (see [3]). Given an n-node plane triangulation $G$, an orderly spanning tree $T$ of $G$ with at most $\left\lfloor\frac{2 n+1}{3}\right\rfloor$ leaves is obtainable in $O(n)$ time.

A floor-plan $F$ of $G$ is a partition of a rectangle into $n$ non-overlapping rectangular modules $r_{1}, r_{2}, \ldots, r_{n}$ such that $v_{i}$ and $v_{j}$ are adjacent in $G$ if and only if the boundaries of $r_{i}$ and $r_{j}$ share at least one non-degenerated line segment. The size of $F$ is the area of the rectangle being partitioned by $F$ with the convention that the corners of all modules are placed on integral grid points. For example, the size of the floor-plan shown in Figure 1(b) is $8 \times 7$.

\section{Our Floor-Planning Algorithm}

This section proves the following main theorem of the paper.

Theorem 1. Given an n-node plane triangulation $G$ with $n \geq 3$, a floor-plan $F$ of $G$ can be constructed in $O(n)$ time such that

1. F consists of I-modules, L-modules, and T-modules only, and

2. the size of $F$ is bounded by $(n-1) \times\left\lfloor\frac{2 n+1}{3}\right\rfloor$. 
Let $T$ be an orderly spanning tree of $G$, where $v_{1}, v_{2}, \ldots, v_{n}$ is the counterclockwise preordering of $T$. Our floor-planning algorithm is described as follows. Pictures of intermediate steps are shown to illustrate how our algorithm obtains the floor-plan in Figure 1(b) for the plane graph $G$ with respect to the orderly spanning tree $T$ shown in Figure 1(a).

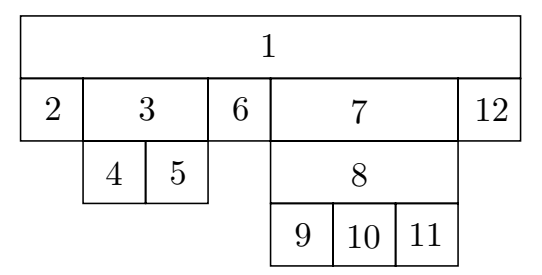

Fig. 3. Step 1: visibility drawing of $T$.

\section{Algorithm FloorPlan $(G, T)$}

Step 1. Produce a (vertical) visibility drawing of $T$ as follows: For each $i=$ $1,2, \ldots, n$, if $v_{i}$ is a leaf of $T$, then draw $v_{i}$ as a unit square; otherwise, draw $v_{i}$ as a $1 \times w(i)$ rectangle. Place each node beneath its parent such that the children of each node is placed in the same order as in $T$.

Comment. For example, Figure 3 shows the resulting visibility drawing for the $T$ shown in Figure 1(a).

Step 2. Turn the above visibility drawing of $T$ into a 2-visibility drawing of $G$ by stretching the nodes downward in the least necessary amount such that $v_{i}$ and $v_{j}$ are horizontally visible to each other if and only if $v_{i}$ and $v_{j}$ is an unrelated edge of $G$ with respect to $T$.

Comment. For example, Figure 4 shows how to obtain the resulting 2visibility drawing for the plane triangulation shown in Figure 1(a). As a matter of fact, this is how Chiang et al. 3 obtained their 2-visibility drawing of $G$ with respect to $T$ with size at most $(n-1) \times w\left(v_{1}\right)$. Note that the resulting drawing still satisfies the property that the bottom boundary of each internal node of $T$ is completely occupied by the top boundaries of its children.

Step 3. First, grow a horizontal branch for $v_{n}$ from boundary of $v_{n}$ visible to $v_{2}$ such that the left boundary of the horizontal branch touches $v_{2}$. Second, for each $i=3,4, \ldots, n-1$, grow horizontal branches for $v_{i}$ from the boundaries of $v_{i}$ visible to $v_{\ell(i)}$ and $v_{r(i)}$ such that the left (respectively, right) boundary of the horizontal branches touches $v_{\ell(i)}$ (respectively, $v_{r(i)}$ ). Furthermore, when extending the boundary of $v_{i}$, we also extend the boundaries of the descendants of $v_{i}$ to maintain the property that the bottom boundary of 


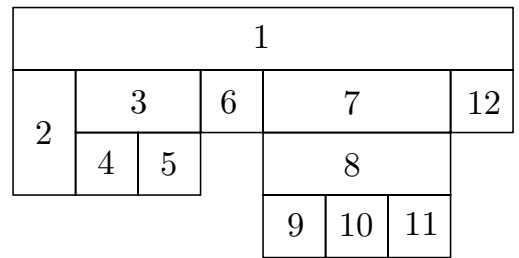

(a)

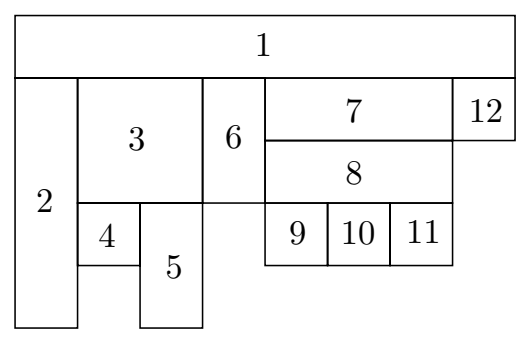

(c)

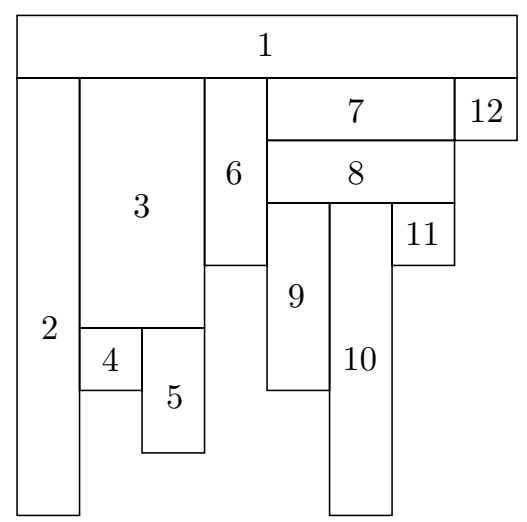

(e)

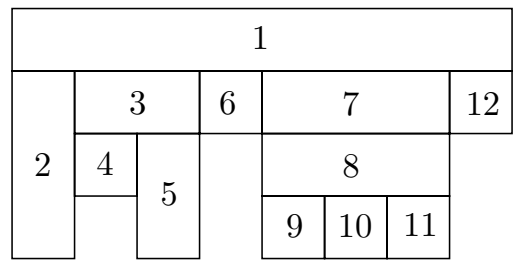

(b)

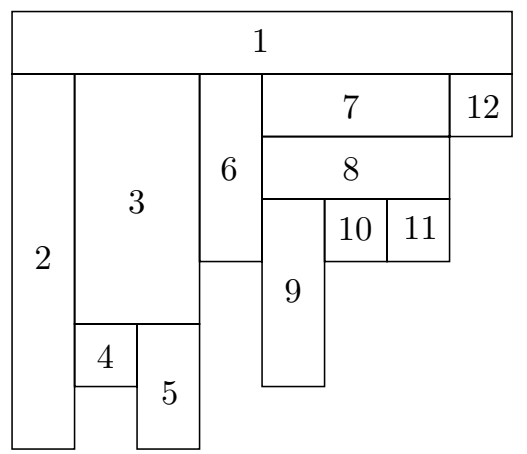

(d)

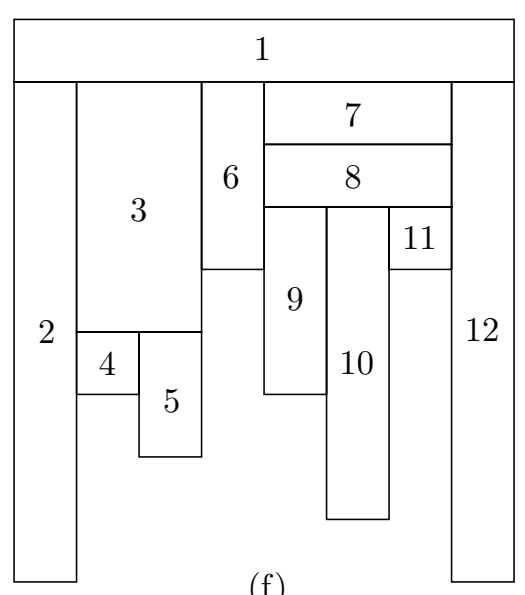

Fig. 4. Step 2: ensuring the horizontal visibility between $v_{i}$ and each node in $B_{2}\left(v_{i}\right)$ for (a) nodes 3 and 4, (b) node 5, (c) nodes 6-8, (d) node 9, (e) node 10, and (f) nodes 11 and 12. 


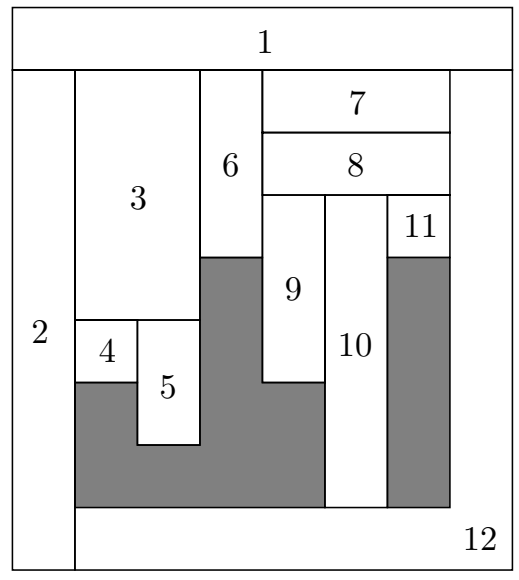

(a)

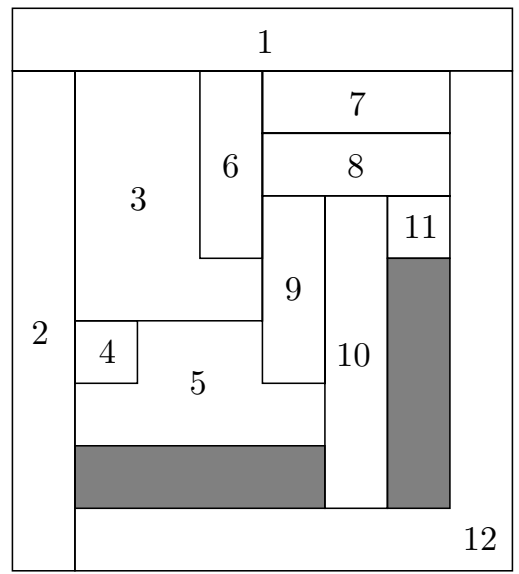

(c)

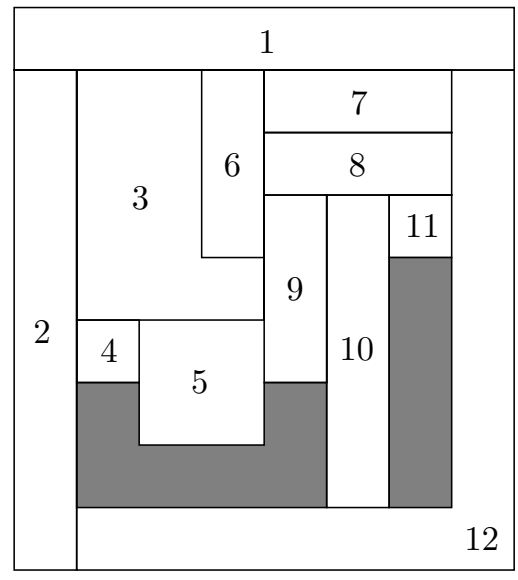

(b)

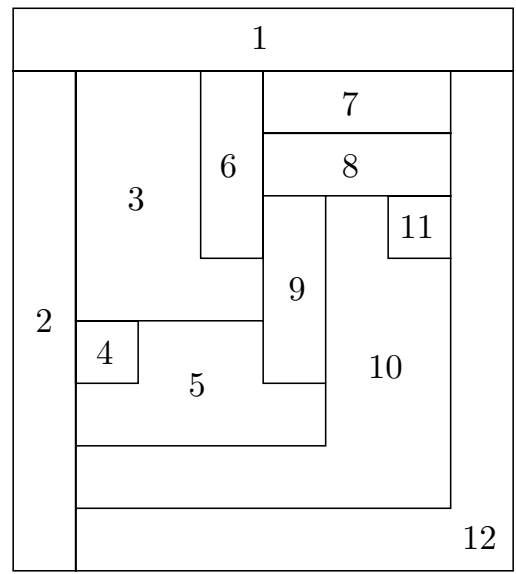

(d)

Fig. 5. Step 3: growing the horizontal branches for (a) node 12, (b) node 3, (c) nodes 4 and 5, and (d) nodes 6-11.

each internal node of $T$ is completely occupied by the top boundaries of its children.

Comment. For example, Figure 5 illustrates how to obtain the resulting drawing for the plane triangulation shown in Figure 1a). Note that when the horizontal branch of node 3 is extended to the right by one unit to touch the left boundary of node 9 , the right boundary of node 5 is also extended to the right by the same amount. 
Step 4. For each $i=n-1, n-2, \ldots, 3$, if $v_{i}$ has a horizontal branch with height greater than one, then reduce the height of the thick branch down to one.

Comment. For example, the module for node 10 in Figure 5(b) has a thick horizontal branch. The height of this thick branch can be reduced by moving down the top boundary of the thick branch that is adjacent to the bottom boundary of node 11 . The resulting floor-plan consists of only I-modules, L-modules, and T-modules. Moreover, the height of each horizontal branch of the L-modules and T-modules is one.

Lemma 2. The following statements hold for our algorithm FLOOR-PLAN.

1. Algorithm FloORPLAN is well defined and can be implemented to run in $O(n)$ time.

2. The output is a floor-plan of $G$ of size no more than $(n-1) \times w\left(v_{1}\right)$.

3. The resulting floor-plan consists of I-modules, L-modules, and T-modules, where the height of each horizontal branch of L-modules and T-modules is one.

Proof. Statement 1 . We verify that each step is well defined and can be implemented to run in $O(n)$ time as follows.

Step 1. Since $w\left(v_{1}\right), w\left(v_{2}\right), \cdots, w\left(v_{n}\right)$ can be computed from $T$ in $O(n)$ time, the described (vertical) visibility drawing of $T$ can easily be computed in $O(n)$ time.

Step 2. Note that we have to ensure that $v_{i}$ and $v_{j}$ are horizontally visible to each other if and only if $v_{j} \in B_{2}\left(v_{i}\right)$ at the end of the stretch-down iteration for $v_{i}$. Therefore, when the boundaries of $v_{i}$ and the nodes in $B_{2}\left(v_{i}\right)$ are stretched down, the boundaries of some other nodes might require being stretched down as well. For example, when we obtain Figure 4(c) from Figure 4(b) by stretching down the boundary of node 6 to ensure that nodes 6 and 8 are horizontally visible to each other, we also have to increase the the heights of nodes 2 and 3 by one. Thus, a naive implementation of this step may require $\Omega\left(n^{2}\right)$ time. However, if we mark the line segments to be stretched down that is to the left of $v_{j}$ and do not actually stretch down the nodes until the completion of the for-loop, then this step can be implemented to run in $O(n)$ time.

Step 3. A naive implementation of this step may require $\Omega\left(n^{2}\right)$ time, since growing the horizontal branches for a node may cause boundary change for its descendants. However, the time complexity can be reduced to $O(n)$ by adapting the "lazy strategy" similar to the one used in the previous step. Since each unrelated edge $\left(v_{i}, v_{j}\right)$ of $G-T$ with $i<j$ and $\left(v_{i}, v_{j}\right) \neq\left(v_{2}, v_{n}\right)$ satisfies exactly one equality of $i=\ell(j)$ and $j=r(i)$, this step is well defined. For the same reason, the resulting drawing is a partition of a rectangle into $n$ rectilinear regions. (That is, there is no gap among modules in the rectangle.) To prove that the resulting drawing is indeed a floor-plan of $G$, it suffices to show that growing 
a horizontal branch of $v_{i}$ is to reach the boundary of $v_{j}$ does not result in new adjacency among these rectilinear modules. Suppose $v_{k}$ is a node whose bottom boundary touches the top bottom of the horizontal branch of $v_{i}$. Assume for a contradiction that $v_{k}$ is not adjacent to $v_{i}$ in $G$. Since the resulting drawing of the previous step is a 2-visibility drawing of $G$, there must be a node $v_{k^{\prime}}$ lies between $v_{i}$ and $v_{k}$ preventing their horizontal visibility to each other. It follows that there is a face of $G$ containing at least four nodes $v_{i}, v_{j}, v_{k}, v_{k^{\prime}}$, contradicting the fact that $G$ is triangulated.

Step 4. By the fact that $T$ is an orderly spanning tree of $G$ and $G$ is a plane triangulation, one can see that if $v_{i}$ grows a horizontal branch to reach $v_{j}$, then there must be a unique node $v_{k}$ whose bottom boundary touches the top boundary of that horizontal branch of $v_{i}$. It is also not difficult to verify that both $\left(v_{i}, v_{k}\right)$ and $\left(v_{j}, v_{k}\right)$ are unrelated edges $G$ with respect to $T$. Thus, in the resulting drawing of the previous step, the left and right boundaries of $v_{k}$ have to touch $v_{i}$ and $v_{j}$. Therefore, the height of that horizontal branch of $v_{i}$ can be reduced to one by moving downward the bottom boundary of $v_{k}$, which is also the top boundary of that horizontal branch, without changing the adjacency of $v_{k}$ to other nodes in the floor-plan. Clearly, each height-reducing operation takes $O(1)$ time, so this step runs in $O(n)$ time. Since the for-loop of this step proceeds from $i=n-1$ down to 3 , each horizontal branch has height exactly one at the end of this step.

Statement 2. As shown by Chiang et al. 3], the resulting drawing of Step 2 is a 2 -visibility of $G$ of size no more than $(n-1) \times w\left(v_{1}\right)$. Since Steps 3 and 4 do not affect the adjacency among the rectilinear modules, the statement is proved.

Statement 3. By the definition of Step 3, one can easily verify that the resulting floor-plan consists of I-modules, L-modules, and T-modules. By the heightreducing operation performed on the horizontal branches in Step 4, the statement is proved.

We are ready to prove the main theorem as follows.

Proof. [for Theorem 1 Straightforward by Lemmas 1 and 2,

\section{Conclusion}

A linear-time algorithm for producing compact floor plans for plane triangulations has been designed, Our algorithm is based upon a newly developed technique of orderly spanning trees with bounded number of leaves [3]. In comparison with previous work on floor-planning plane triangulations [9], our algorithm is simpler in the algorithm itself as well as in the resulting floor-plan in the sense that the Z-modules required by [9] is not needed in our design. Another important feature of algorithm algorithm is the upper bound $(n-1) \times\left\lfloor\frac{2 n+1}{3}\right\rfloor$ on the area of the output floor-plan. Previous work [921] does not provide any area bounds on their outputs. Investigating whether the $(n-1) \times\left\lfloor\frac{2 n+1}{3}\right\rfloor$ area is worst-case optimal is an interesting future research direction. Another interesting 
possibility is modifying our floor-plan algorithm into an algorithm to compute rectangular-dual with bounded area for plane triangulation without separating triangles.

\section{References}

1. J. Bhasker and S. Sahni. A linear algorithm to check for the existence of a rectangular dual of a planar triangulated graph. Networks, 17:307-317, 1987.

2. J. Bhasker and S. Sahni. A linear algorithm to find a rectangular dual of a planar triangulated graph. Algorithmica, 3:247-278, 1988.

3. Y.-T. Chiang, C.-C. Lin, and H.-I. Lu. Orderly spanning trees with applications to graph encoding and graph drawing. In Proceedings of the 12th Annual ACM-SIAM Symposium on Discrete Algorithms, pages 506-515, Washington, D. C., USA, 7-9 Jan. 2001. A revised and extended version can be found at http://xxx.lanl.gov/abs/cs.DS/0102006.

4. R. C.-N. Chuang, A. Garg, X. He, M.-Y. Kao, and H.-I. Lu. Compact encodings of planar graphs via canonical ordering and multiple parentheses. In K. G. Larsen, S. Skyum, and G. Winskel, editors, Proceedings of the 25th International Colloquium on Automata, Languages, and Programming, Lecture Notes in Computer Science 1443, pages 118-129, Aalborg, Denmark, 1998. Springer-Verlag.

5. H. de Fraysseix, P. Ossona de Mendez, and P. Rosenstiehl. On triangle contact graphs. Combinatorics, Probability and Computing, 3:233-246, 1994.

6. H. de Fraysseix, J. Pach, and R. Pollack. How to draw a planar graph on a grid. Combinatorica, 10:41-51, 1990.

7. U. Fößmeier, G. Kant, and M. Kaufmann. 2-visibility drawings of planar graphs. In S. North, editor, Proceedings of the 4th International Symposium on Graph Drawing, Lecture Notes in Computer Science 1190, pages 155-168, California, USA, 1996. Springer-Verlag.

8. X. He. On finding the rectangular duals of planar triangular graphs. SIAM Journal on Computing, 22:1218-1226, 1993.

9. X. He. On floor-plan of plane graphs. SIAM Journal on Computing, 28(6):21502167, 1999.

10. G. Jacobson. Space-efficient static trees and graphs. In Proceedings of the 30th Annual Symposium on Foundations of Computer Science, pages 549-554, Research Triangle Park, North Carolina, 30 Oct.-1 Nov. 1989. IEEE.

11. G. Kant. Drawing planar graphs using the canonical ordering. Algorithmica, 16(1):4-32, 1996.

12. G. Kant and X. He. Regular edge labeling of 4-connected plane graphs and its applications in graph drawing problems. Theoretical Computer Science, 172(12):175-193, 1997.

13. K. Koźmiński and E. Kinnen. Rectangular duals of planar graphs. Networks, 15(2):145-157, 1985.

14. K. A. Kózmiński and E. Kinnen. Rectangular dualization and rectangular dissections. IEEE Transactions on Circuits and Systems, 35(11):1401-1416, 1988.

15. Y. T. Lai and S. M. Leinwand. A theory of rectangular dual graphs. Algorithmica, 5(4):467-483, 1990.

16. K. Mailing, S. H. Mueller, and W. R. Heller. On finding most optimal rectangular package plans. In Proceedings of the 19th Annual IEEE Design Automation Conference, pages 263-270, 1982. 
17. J. I. Munro and V. Raman. Succinct representation of balanced parentheses, static trees and planar graphs. In Proceedings of the 38th Annual Symposium on Foundations of Computer Science, pages 118-126, Miami Beach, Florida, 20-22 Oct. 1997. IEEE.

18. W. Schnyder. Planar graphs and poset dimension. Order, 5:323-343, 1989.

19. W. Schnyder. Embedding planar graphs on the grid. In Proceedings of the First Annual ACM-SIAM Symposium on Discrete Algorithms, pages 138-148, 1990.

20. S. Tsukiyama, K. Koike, and I. Shirakawa. An algorithm to eliminate all complex triangles in a maximal planar graph for use in VLSI floorplan. In Proceedings of the IEEE International Symposium on Circuits and Systems, pages 321-324, 1986.

21. K.-H. Yeap and M. Sarrafzadeh. Floor-planning by graph dualization: 2-concave rectilinear modules. SIAM Journal on Computing, 22(3):500-526, 1993. 